\title{
Characterization of Viscoelastic Materials Using Free-Layered and Sandwiched Samples: Assessment and Recommendations
}

\author{
M. S. Ozer ${ }^{a *}$, H. KORUK ${ }^{b}$, K. Y. SANLITURK ${ }^{a}$ \\ ${ }^{a}$ Istanbul Technical University, Mechanical Engineering Department, 34437 Istanbul, Turkey \\ ${ }^{b}$ MEF University, Mechanical Engineering Department, 34396 Istanbul, Turkey
}

\begin{abstract}
Viscoelastic materials are widely used in many applications in practice. However, determination of the elastic and damping properties of these materials is quite difficult in the sense that the identified results may contain high degree of uncertainty. The characterization of viscoelastic materials using the Oberst beam method, based on non-contact excitation and response measurements, is revisited in this paper. The effects of signal processing parameters such as frequency resolution in Frequency Response Function (FRF) measurements, as well as the effects of various single-degree-of-freedom modal analysis methods, including circle-fit, half-power and line-fit are investigated first. Then, the modal loss factors, Young's modulus and shear modulus of some sample viscoelastic materials are identified using both the free-layered and sandwiched samples. The results obtained from different tests are compared, discussed and some recommendations are made so as to identify the damping and elastic properties of typical viscoelastic materials with better accuracy. Analyses of a large number of FRF measurements show that the selection of the appropriate signal processing parameters and the use of appropriate modal analysis method can be very significant during the identification of viscoelastic materials. By following the approach presented in this paper, the damping and elastic properties of viscoelastic materials can be identified with better accuracy using either free-layered or sandwiched samples. The material properties obtained by this approach can be used for developing valid structural models and/or for damping optimization purposes.
\end{abstract}

DOI: 10.12693/APhysPolA.127.1251

PACS: $46.35 .+\mathrm{z}, 46.80 .+\mathrm{j}$

\section{Introduction}

Viscoelastic materials are widely used in many applications in practice $[1,2]$. However, determination of the elastic and damping properties of these materials is quite difficult in the sense that the identified material properties may contain high degree of uncertainty. The Oberst Beam Method (OBM) based on non-contact excitation and response measurements is widely used for the characterization of viscoelastic materials [1]. However, the effects of signal processing parameters such as frequency resolution in Frequency Response Function (FRF) measurements, as well as the effects of selected modal analysis method and the difficulties in dealing with various types of test samples, should be investigated in order to obtain accurate material properties.

In the OBM, the FRF measurements are conducted using both bare and composite beams. The natural frequencies of the bare beam in the frequency range of interest are first identified by analyzing the FRF measured for the bare sample. Then, the measured FRF of the composite beam is analyzed in order to determine the natural frequencies and modal loss factors of the composite beam. Young's/shear moduli and damping levels (loss factors) of the damping materials, corresponding to the individual vibration modes, are then extracted.

It should be noted that although the OBM is well

* corresponding author; e-mail: ozermehmet1@itu.edu.tr known for a long period of time, performing a successful characterization of material properties using the OBM is not an easy task. The effects of various parameters including the adverse effects of non-contact excitation system and the length of the test specimen were examined in the past $[3,4]$. In this study, the effects of signal processing parameters such as frequency resolution in FRF measurements and the selected modal analysis methods, including circle-fit, half-power and line-fit for modal identification are investigated. Then, the Young's/shear moduli and modal loss factors of some sample viscoelastic materials are identified using various types of test samples.

\section{Effects of signal processing parameters}

The frequency range during the tests was set to $f=2-2000 \mathrm{~Hz}$ and the beams are excited using a random signal. Hanning windowing function is applied to both response and excitation signals. The length of test samples is selected as $L=205 \mathrm{~mm}$. The width and thickness of both bare (steel) and damping layers (Damping Material-1, DM-1) are $w=10 \mathrm{~mm}$ and $h=1 \mathrm{~mm}$. The densities of the bare beam and the damping material are $\rho=7900 \mathrm{~kg} / \mathrm{m}^{3}$ and $\rho_{d m}=1953 \mathrm{~kg} / \mathrm{m}^{3}$, respectively. The length of the beam section, exposed to the electromagnetic field, is selected based on the recommendations presented in previous studies $[4,5]$. Overall, the length of the beam exposed to the electromagnetic excitation is approximately $\delta=2.5 \mathrm{~mm}$. The distance of the response transducer from the root of the beam is $a=33 \mathrm{~mm}$. A number of averaged redings should be performed to minimize noise levels of the signals. Some 
preliminary tests revealed that acceptable results can be using 10 or more measurements. Here, 30 readings were used for averaging during spectrum measurements. In order to determine other signal processing parameters such as frequency resolution and the method of system identification, a large number of FRFs are measured using several samples under various conditions. A steel beam, two 'identical' one-sided (free-layered) damped beams, a double-sided (free-layered) damped beam and a few sandwiched (constrained-layered) beams are tested. The FRF measurements are conducted at four different frequency resolutions (i.e., $\Delta f=0.3125,0.625,1.25$ and $2.5 \mathrm{~Hz}$ ). The so called half-power, circle-fit and line-fit methods $[6,7]$ are used to extract the modal parameters from measured FRFs.

Three sets of FRF measurements at each frequency resolution are conducted for each test sample and they are analyzed using the aforementioned three methods. It should be noted that the effect of the frequency resolution on the accuracy of natural frequencies was already investigated in the literature and the three methods predict almost the same natural frequency values for a given frequency resolution. However, the effect of frequency resolution is found to be much more critical when damping levels are to be identified. Therefore, the effects of frequency resolution and modal analysis method on identified modal loss factors are investigated in detail here. Overall, the average values of the modal loss factors of a bare (steel) beam, one-sided damped beam, a double-sided damped beam and sandwiched beam are determined using three FRFs and three modal analysis methods for four frequency resolution values and the results are presented in Fig. 1. It is seen that, in general, higher loss factors are obtained when the frequency resolution values are higher (coarser).
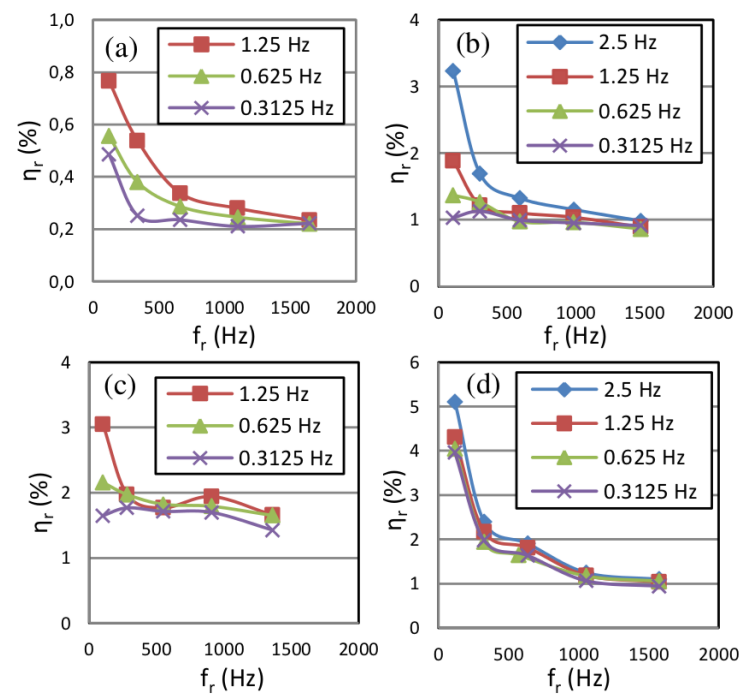

Fig. 1. Identified modal loss factors of various test sample configurations for different values of frequency resolution: bare-steel (a), one-sided damped (b), double-sided damped (c) and (d) sandwich-1.
The results show that the modal loss factors of the bare (Fig. 1a), one-sided damped (Fig. 1b), double-sided damped (Fig. 1c) and sandwich (Fig. 1d) samples converge to the same values as natural frequency (or mode number) increases, for all frequency resolutions. The modal loss factors of the bare (steel) and double-sided damped samples identified using the half-power (HP), circle-fit (CF) and line-fit (LF) methods, as a function of frequency for three different values of frequency resolution are presented in Fig. 2a and Fig. 2b, respectively. It is obvious that convergence of modal loss factor is related to frequency resolution. It is worth noting that the loss factor of the steel material is obtained to be about $0.2 \%$ $[1,3]$ when tests are performed in ambient conditions. The results for the bare steel beam in Fig. 2a show that a level of damping close to $0.2 \%$ is identified for all the modes and for all values of frequency resolution as natural frequency (or mode number) increases; the refining of the frequency resolution leads to convergence of the modal damping levels to their "correct" values. It is also interesting that the performance of the line-fit method is better than of the others, even at the moderate (or coarse) values of frequency resolution. However, it is seen that the half-power method that takes into account only the amplitudes and the circle-fit method can be used for damping identification purposes, as long as the frequency resolution is sufficiently fine enough.
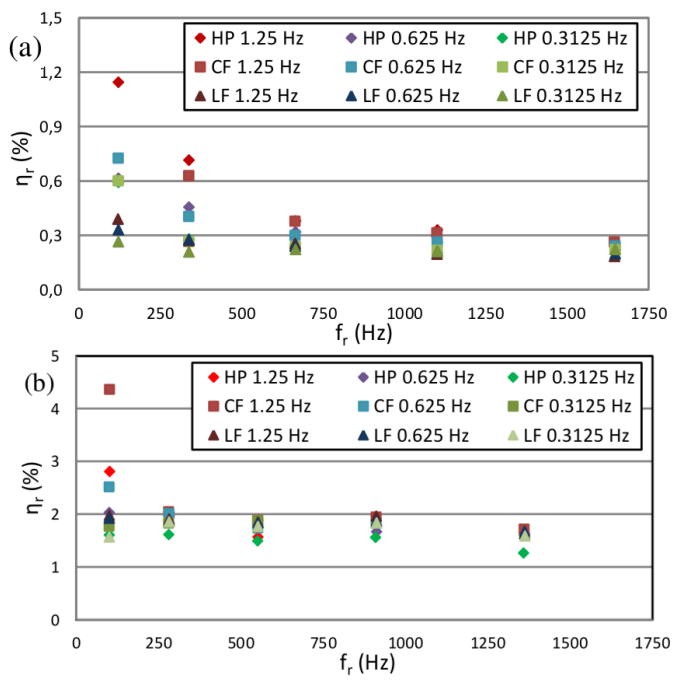

Fig. 2. Identified modal loss factors of the steel (a) and double-sided damped (b) samples.

Here, a normalized frequency resolution parameter $\left(\Delta f / f_{r}\right)$ is defined, so as to highlight the effect of frequency resolution. The modal damping results for the steel and double-sided damped samples are presented in Fig. 3 using this normalized resolution parameter. It is seen that the line-fit method gives consistent results even at very high $\Delta f / f_{r}$ values, whereas the deviations in the identified modal loss factors predicted using the circle-fit and half-power methods can be quite high, when $\Delta f / f_{r}>0.01$ for both the steel (Fig. 3a) and damped (Fig. 3b) beams. 

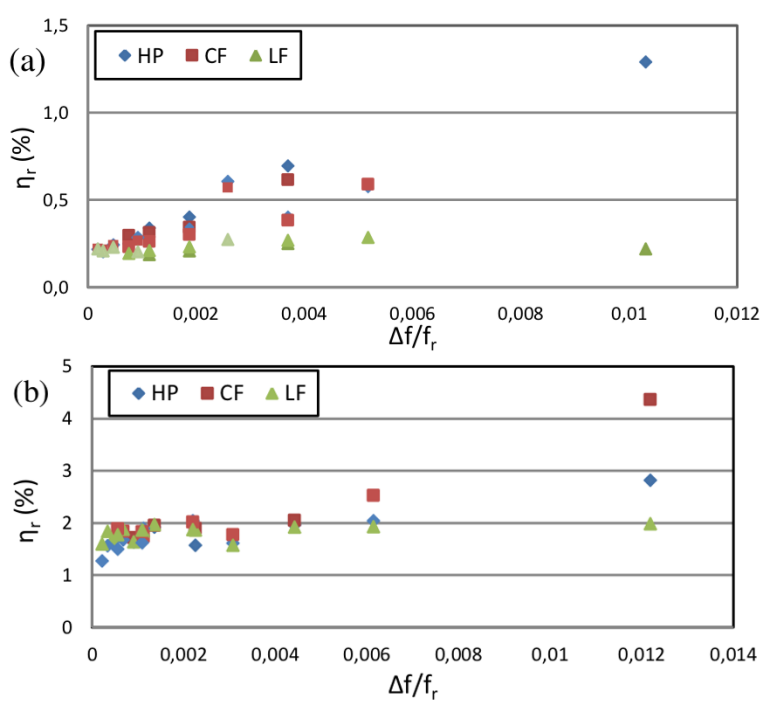

Fig. 3. Identified modal loss factors of the steel (a) and double-sided damped (b) samples.

\section{Identification of material properties}

Once the natural frequencies and loss factors of the bare and the damped samples are identified using measured FRFs, the Young moduli $\left(E_{d m}\right)$ and/or shear moduli $\left(G_{d m}\right)$ and loss factors $\left(\eta_{d m}\right)$ of the damping material for the individual modes are estimated. Here, the natural frequencies and modal loss factors identified via the finer values of frequency resolution for individual test samples and the line-fit method are used to extract material properties. For a double-sided damped specimen, the Young's modulus and loss factor of the damping materials are determined by [1]:

$$
\begin{aligned}
& E_{d m}=E \frac{\left[\left(f_{m} / f_{n}\right)^{2}(1+2 D T)-1\right]}{\left(8 T^{3}+12 T^{2}+6 T\right)}, \\
& \eta_{d m}=\eta_{m}+\left[\frac{E \eta_{m}}{E_{d m}\left(8 T^{3}+12 T^{2}+6 T\right)}\right],
\end{aligned}
$$

where $E$ is Young's modulus of bare beam, $f_{m}$ and $f_{n}$ are resonant frequencies of the composite and bare beams, respectively, $D$ is the density ratio, $T$ is the thickness ratio and $\eta_{m}$ is the loss factor of the composite beam. Results show that the one-sided damped beam did not provide meaningful material properties as the precautions in the standard [1] could not be satisfied. The material properties of DM-1, identified using the double-sided damped beam are listed in Table. It is worth stating that material properties of DM-1 are also aimed to be identified using the measurement of sandwiched specimen. However, it was not possible to obtain reliable and meaningful results using the sandwiched specimen (shear moduli values were much lower than expected), hence they are not presented here. The reason for this problem might be related to the way the damping material was glued to the base beam, a problem which needs further investigation. The material properties of another material named as Damping
Material-2 (DM-2) with thickness $h=2 \mathrm{~mm}$ and density $\rho_{d m}=1460 \mathrm{~kg} / \mathrm{m}^{3}$ are also aimed to be identified using a one-sided damped beam and a sandwiched specimen. The properties obtained using the free-layered specimen are also listed in Table. Although the measured FRFs are not presented here for brevity, it was noticed that the level of damping for the sandwich specimen with DM-2 was so high that it was not possible to identify the vibration modes and corresponding natural frequencies and modal damping values. The results in this study show that the mechanical properties of viscoelastic materials can be identified with sufficient level of confidence using free-layered samples. However, there are significant difficulties when sandwiched specimens are to be used for similar purposes.

Identified Young's moduli and loss factors of Damping Material-1 and

TABLE

\begin{tabular}{|c|c|c|c|}
\hline \multicolumn{4}{|c|}{ Double-sided damped with Damping Material-1 } \\
\hline Mode No & $f_{r}[\mathrm{~Hz}]$ & $E_{d m}[\mathrm{MPa}]$ & $\eta_{d m}[\%]$ \\
\hline 2 & 101.55 & 433.7 & 30.53 \\
\hline 3 & 282.84 & 346.1 & 43.15 \\
\hline 4 & 552.69 & 253.0 & 57.75 \\
\hline 5 & 912.87 & 203.2 & 72.90 \\
\hline 6 & 1362.61 & 167.9 & 79.17 \\
\hline \multicolumn{4}{|c|}{ One-sided damped with Damping Material-2 } \\
\hline Mode No & $f_{r}[\mathrm{~Hz}]$ & $E_{d m}[\mathrm{MPa}]$ & $\eta_{d m}[\%]$ \\
\hline 2 & 122.59 & 1109.6 & 37.51 \\
\hline 3 & 346.64 & 1189.3 & 40.88 \\
\hline 4 & 689.36 & 1301.3 & 46.10 \\
\hline
\end{tabular}
Damping Material-2.

\section{Conclusions}

In this paper, the characterization of viscoelastic materials using the Oberst beam method is examined. The effects of some signal processing parameters such as frequency resolution, as well as modal analysis methods including half power, circle-fit and line-fit methods are investigated. Both the free-layered and sandwiched samples are used for the purpose of identifying material properties.

The results show that the frequency resolution is one of the critical parameter for identification of modal loss factors. It is seen that, in general, higher loss factors are obtained when the frequency resolution is not fine enough. It is also noted that estimated modal loss factors for the lower modes are more adversely affected by inadequate frequency resolution. One can see that modal loss factor analyzed with different frequency resolution converge to the same levels as the mode number increases. It is strongly recommended that some trial tests should be conducted first, so as to identify the appropriate frequency resolution in FRF measurements for damping estimation purposes. If necessary, two or more frequency resolutions can be used; the finer one for the lower modes and the coarser one for the higher modes. 
The results also indicate that the performance of the line-fit method is better than those of other methods considered here, even at moderately coarse values of frequency resolutions. However, if the frequency resolution is guaranteed to be fine enough for all the modes within the frequency range of interest, the half-power method or the circle-fit method can also be used. The results obtained by analyzing a large number of FRFs show that the selection of appropriate signal processing parameters and the use of the appropriate modal analysis method are crucial during the identification of the properties of viscoelastic materials. Finally, it is noted that the material properties can be identified with high level of confidence using free-layered samples. Identification of material properties using sandwiched specimens may lead to significant difficulties in practice.

\section{References}

[1] ASTM E756-05, Standard Test Method for Measuring Vibration-Damping Properties of Materials, American Society for Testing Materials, 2005.
[2] SAE J1637, Laboratory Measurement of the Composite Vibration Damping Properties of Materials on a Supporting Steel Bar, SAE International, 1993.

[3] H. Koruk, K.Y. Sanliturk, On Measuring Dynamic Properties of Damping Materials Using Oberst Beam Method, ASME $201010^{\text {th }}$ Biennial Conference on Engineering Systems Design and Analysis, Istanbul, Turkey, 2010.

[4] H. Koruk, K.Y. Sanliturk, Mech. Syst. Signal Pr. 30, 274 (2012).

[5] H. Koruk, K.Y. Sanliturk, Modelling Electromagnetic Effect of The Non-Contact Excitation System in Oberst Beam Method, Inter-Noise 2013: The 42nd International Congress and Exposition on Noise Control Engineering, Paper no: 1261, Innsbruck, Austria, 15-18 September, 2013.

[6] D.J. Ewins, Modal Testing: Theory, Practice and Application, Second Edition, Research Studies Press, Hertfordshire, 2000.

[7] ICATS: Imperial College Testing Analysis and Software, Imperial Collage London, Dynamic Section, London, 2009. 